\title{
A Tabu Search Based Solution Approach to the Competitive Multiple Allocation Hub Location Problem
}

\author{
N. Ghaffarinasab'*, Y. Jabarzadeh', A. Motallebzadeh
}

The hub location problems (HLP) constitute an important class of facility location problems that have been addressed by numerous operations researchers in recent years. HLP is a strategic problem frequently encountered in designing logistics and transportation networks. Here, we address the competitive multiple allocation HLP in a duopoly market. It is assumed that an incumbent firm (the leader) is operating an existing hub network in a market and an entrant firm (the follower) tries to enter the market by locating its own hubs aiming at capturing as much flow as possible from the leader. The customers choose one firm based on the service level (cost, time, distance, etc.) provided by the firm. We formulate the problem from the entrant firm's point of view and propose an efficient tabu search based solution algorithm to solve it. Computational experiments show the capability of the proposed solution algorithm to obtain the optimal solutions in short computing times.

Keywords: Hub location, Competitive models, Mathematical formulation, Tabu search.

Manuscript was received on 01/06/2017, revised on 23/09/2017 and accepted for publication on 25/09/2017.

\section{Introduction}

Hub networks play major roles in reducing costs and enhancing service levels in many transportation, telecommunications, and computer networks. These networks provide efficient services between many origins-destination $(\mathrm{O} / \mathrm{D})$ pairs of nodes via a set of hubs that serve as switching and flow consolidation points. The hub location problem (HLP) deals with determining the location of hub facilities and allocation of non-hub nodes (spokes) to the installed hubs in such a way that the traffic between O/D pairs be routed in a most favorable way [1].

The non-hub nodes can be allocated to the hubs based on one of the two basic allocation types called the single and multiple allocation. In a single allocation network, all the incoming and outgoing traffic to/from each demand node is routed through a single hub, whereas in a multiple allocation network, every demand node can receive and send flow via more than one hub. An example of a multiple allocation hub-and-spoke network which is the underlying network topology our investigation is shown in Fig. 1.

\footnotetext{
* Corresponding Author.

${ }^{1}$ Department of Industrial Engineering, University of Tabriz, Tabriz, Iran, Email: ngnasab@tabrizu.ac.ir.

2 Faculty of Economics, Management and Business, University of Tabriz, Tabriz, Iran, Email: yjabarzade@tabrizu.ac.ir.

3 Faculty of Economics, Management and Business, University of Tabriz, Tabriz, Iran, Email: ar.motallebzadeh@yahoo.com.
} 


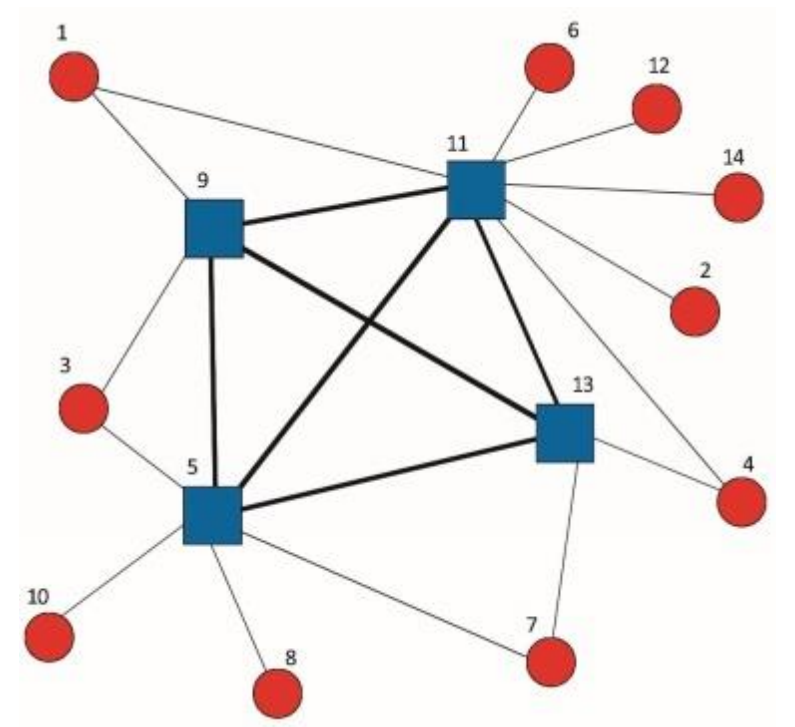

Figure 1. Example of a multiple allocation hub network

Most studies in the literature of HLP deal with situations in which the markets are monopolistic and try to model and solve the problems on behalf of a firm operating in such a market. However, in real world applications, there may be competitors present in the market whose decisions would definitely affect the level of goal achievement for the other firms. In here, we address the competitive multiple allocation HLP in a duopoly market. We assume that an incumbent firm (the leader) is operating an existing hub network in a market and an entrant firm (the follower) tries to enter the market by configuring its own hub network trying to capture as much flow as possible from the leader. The customers choose one firm based on the service level (cost, time, distance, etc.) provided by the firm. We consider the follower's problem which aims at optimally locating its hubs, based on the known decisions already made by leader. To this end, a mixed integer programming (MIP) model is developed for the follower's problem and an efficient solution procedure based on Tabu Search (TS) metaheuristic is proposed to solve the problem.

The remainder of our work is organized as follows. The next section briefly reviews the relevant literature to the problem. In Section 3, we will present a mathematical formulation of the problem. Our proposed TS algorithm is presented in Section 4. Computational experiments and corresponding results are presented in Section 5. Finally, our concluding remarks and directions for future works are given in Section 6.

\section{Literature Review}

HLP was first addressed by O'Kelly [20]. He presented the first mathematical formulation and solution method for the single allocation HLP. Later, O'Kelly [21] developed the first quadratic mathematical formulation for the uncapacitated single allocation $p$-hub median problem (USA $p$ HMP). Campbell [2] proposed linear integer programming formulations for different versions of HLP such as $p$-hub median problem, the uncapacitated hub location problem, $p$-hub center problem, and hub covering problem both in single and multiple allocation settings.

The $p$-hub maximal covering problem is a variant of hub covering problem where the objective is to maximize the covered flow in a hub network using a fixed number of hubs. Originally, $p$-hub 
covering problem was modeled by Campbell [2]. Kara and Tansel [13] proposed new mathematical formulations for the single allocation hub covering problem. Wagner [31] proposed the hub covering location problem with bounded path lengths. Yildiz and Karaşan [32] applied the hub covering concept to model the regenerator location problem (RLP) in telecommunication networks and proposed a branch and cut algorithm for solving their model. Jabalameli et al. [12] proposed an efficient simulated annealing based heuristic for solving the single allocation $p$-hub maximal covering problem. Pasandideh et al. [22] proposed a bi-objective hub maximal covering problem considering time-dependent reliabilities. Karimi et al. [14] formulated the multi-modal single allocation capacitated $p$-hub covering problem over fully interconnected hub networks. Peker and Kara [23] proposed efficient mathematical formulations for the $p$-hub maximal covering problem with both single and multiple assignments. The authors also extended their models to the case of partial coverage. For more details on HLP and recent advances in this field, the interested readers are referred to surveys given in [1], [3], and [9].

The hub location problem with competition was first addressed by Marianov et al. [18]. Given a set of existing hubs for the leader, they formulated the follower's problem as maximization of its market share. Their model allows partial captures by the follower depending on the provided service levels. Wagner [30] addressed for a similar problem with a different capture paradigm where the follower gets nothing in case of equal service level for the same problem. In another competitive HLP, an entrant airline transportation firm was assumed to enter a competitive market [7]. It was also assumed that the customers choose an airline based on a combination of factors such as flying time and travel fare based on gravity like utility functions. Lin and Lee [15] studied a competition game on hub network design and determined a hub network for each one of the carriers in the oligopolistic market based on the long-term Cournot-Nash equilibrium steady state. Lüer-Villagra and Marianov [16] addressed a competitive HLP where location and pricing decisions were made by an entrant firm entering into a market where some other firms had already been operating. Customer preferences were modeled using a logit function resulting in a nonlinear model maximizing the profit of the entrant firm. Another research conducted by Sasaki et al. [24] deals with a competitive hub arc location problem under the Stackelberg competition. In their problem, rather than locating hub facilities, hub arcs are located in the network. It is also assumed that the capture of demands by the players follows a gradual capture mechanism rather than a binary all-or-nothing capture mechanism. To maximize their own revenues, the leader and the follower locate $p$ and $r$ hub arcs, respectively. They modeled the problem as a bilevel program. Čvokić et al. [5] considered a competitive market where prices had fixed markups and the each one two competitors (a leader and a follower) try to maximize his/her profit. The aim of this problem is to find the optimal hub and spoke network for the leader. They formulated the problem as a bilevel nonlinear MIP problem and proposed a heuristic as a solution approach. Furthermore, Čvokić et al. [6] demonstrated the existence of the unique Stackelberg and Nash pricing equilibria by proposing a model where two competitors (the leader and follower) are sequentially creating their hub and networks and setting prices. A new hub-and-center transportation network problem for a new company competing against an operating company was introduced by Niknamfar et al. [19]. In their research, a new company aims to maximize the total captured flow and to minimize the total transportation cost by locating $p$ hubs and assigning the center nodes. They proposed two multi-objective meta-heuristic algorithms (MOBBO and NSGAII) to solve this NP-hard problem.

In another study, Mahmutogullari and Kara [17] considered a competitive multiple allocation HLP based on the Stackelberg competition where the market was assumed to be a duopoly. Two firms decide locations of their hubs and then customers choose one firm with respect to provided service levels. They named the follower's problem as the $\left(r \mid X_{p}\right)$ hub-medianoid and the leader's problem as the $(r \mid p)$ hub-centroid problem. Both problems were formulated as MIP models and exact solution 
algorithms based on enumeration were proposed for solving them. More recently, Ghaffarinasab et al. [10] addressed the competitive single and multiple allocation HLPs as Stackelberg games and proposed efficient simulated annealing (SA) based heuristics for solving the problems in both single level (follower's problem) and bilevel (leader's problem) settings.

Although integer programming optimization approaches are used to solve small hub problems, large instances of the HLP are usually solved by heuristic or metaheuristic procedures as the HLP belongs to the class of hard combinatorial optimization problems. The main advantage of the metaheuristic algorithms is that they obtain optimal or near-optimal solutions in relatively short computing times. For this reason, metaheuristic approaches are being extensively used in practice for many real world applications. Many researchers have already addressed different variants of the HLP using metaheuristic algorithms. A tabu search (TS) heuristic was proposed for the uncapacitated single allocation $p$-hub median problem [27]. Ernst and Krishnamoorthy [8] developed an SA heuristic for the same problem and showed that it was comparable, in both solution quality and computing time, to the TS heuristic [27]. Topcuoglu et al. [29] proposed a GA algorithm for the uncapacitated single allocation hub location problem (USAHLP). Another hybrid heuristic for this problem was proposed by Chen [4]. His hybrid heuristic outperformed the heuristic of [29] both in solution time and quality. Silva and Cunha [25] proposed three variants of a simple and efficient multi-start TS heuristic as well as a two-stage integrated TS heuristic to solve the uncapacitated single allocation HLP. In another work, Silva and Cunha [26] proposed a TS heuristic for the model of uncapacitated single allocation $p$-hub maximal covering problem.

Here, we consider the competitive hub location problem stated in [17] from the follower's perspective. A new mathematical programming formulation based on the concept of maximal covering HLP is proposed for the problem. To solve the proposed model, an efficient tabu search based solution procedure is developed having the capability to solve the problem to optimality in a short computational time.

\section{Mathematical Formulation}

Assume that $G=(N, E)$ is a graph in which $N$ is the set of nodes and $E$ is the set of edges $(E \subseteq$ $N \times N)$. Let $H \subseteq N$ be a subset of the nodes that are candidate for opening hubs. Also, for all $i, j \in$ $N$, let $w_{i j}$ and $c_{i j}$ respectively denote the amount of flow originated at node $i$ and destined to node $j$, and the transportation cost of a unit flow from node $i$ to node $j$. Transportation costs on inter-hub connections are discounted by a constant factor $\alpha(0 \leq \alpha \leq 1)$ to reflect the scale economies on connections between the hubs. It is assumed that the leader is operating its network of $p$ hubs and the follower wants to enter the market. The number of hubs to be located by the follower is $r$. Customers are captured by the leader or follower based on the respective provided service levels. Service level is defined as the cost of routing a unit flow on the route from its origin to its destination. A customer prefers the follower if the service level provided by the follower is strictly better than that of the leader, otherwise the demand is captured by the leader. In case of equal service levels, ties are broken in favor of the leader as the customer has no incentive to change the current position. The notations and definitions used in the remainder of this section are mostly borrowed from [17] as we address similar problems and also we want the terminology to be consistent in the competitive hub location literature. 
Let us assume that the leader has already opened her hubs at a subset of nodes $X_{p}=$ $\left\{x_{1}, x_{2}, \ldots, x_{p}\right\}, X_{p} \subseteq H$ and is serving the market with these hubs. For every node pair $i$ and $j$, the service level provided by the leader, denoted by $\beta_{i j}$, can easily be calculated by [17]:

$$
\beta_{i j}=\min _{k, m \in X_{p}}\left\{c_{i k}+\alpha c_{k m}+c_{m j}\right\}, \quad \forall i, j \in 0
$$

Assume now that the follower enters the market and establishes her hubs on a subset of nodes $Y_{p}=\left\{y_{1}, y_{2}, \ldots, y_{r}\right\}, Y_{r} \subseteq H$. In a similar manner, the follower's service levels, denoted by $\gamma_{i j}$, for all node pairs $i$ and $j$ can be calculated as [17]:

$$
\gamma_{i j}=\min _{k, m \in Y_{r}}\left\{c_{i k}+\alpha c_{k m}+c_{m j}\right\}, \quad \forall i, j \in 0
$$

For all $i, j \in N$, the follower captures the flow $w_{i j}$ if $\gamma_{i j}<\beta_{i j}$. Therefore, the total flow captured by the follower can be expressed by a mapping $f: P_{p}(H) \times P_{r}(H) \rightarrow[0, W]$ such that:

$$
f\left(X_{p}, Y_{r}\right)=\sum_{i, j \in N: Y_{i j}<\beta_{i j}} w_{i j}
$$

where $P_{p}(H)$ is the set of all subsets of cardinality $p$ from $H$ and $W$ is the sum of flows over the network, that is,

$$
W=\sum_{i, j \in N} w_{i j}
$$

Given the leader's hubs located on $X_{p}$, the follower's problem is to locate a set of $r$ hubs that maximizes the captured demand by her. To model this problem, assume that $q_{i j k m}$ is a binary covering parameter that takes the value of 1 if the flow between nodes $i$ and $j$ is captured by the follower and 0 , otherwise. In other words, with $\beta_{i j}$ defined by (1) for a fixed $X_{p}$,

$$
q_{i j k m}=\left\{\begin{array}{ll}
1, & \text { if } c_{i k}+\alpha c_{k m}+c_{m j}<\beta_{i j}, \\
0, & \text { otherwise, }
\end{array} \quad \forall i, j \in N, \forall k, m \in 0\right.
$$

Let the variable $z_{i j k m}$ denote the fraction of flow $w_{i j}$ that is sent from node $i$ to node $j$ using the link between the hubs $k$ and $m$ by the follower. Let also the binary variable $y_{k} \in\{0,1\}$ be 1 if node $k$ is selected by the follower as a hub and 0 , otherwise. The problem consists of the selection of nodes which will act as the follower's hubs and determining how the non-hub nodes will be allocated to the hub nodes and the flows will be routed in the network so that the total captured flow by the follower is maximized. The MIP model for the follower's problem can now be written as follows:

$$
\begin{array}{ll}
\max & \sum_{i \in N} \sum_{j \in N} \sum_{k \in H} \sum_{m \in H} w_{i j} q_{i j k m} z_{i j k m} \\
\text { s.t. } & \sum_{k \in H} y_{k}=r \\
& \sum_{k \in N} \sum_{m \in H} z_{i j k m}=1, \quad \forall i, j \in N
\end{array}
$$




$$
\begin{array}{ll}
\sum_{m \in H} z_{i j k m}+\sum_{m \in H \mid m \neq k} z_{i j m k} \leq y_{k}, & \forall i, j \in N, k \in H \\
z_{i j k m} \geq 0, & \forall i, j \in N, k, m \in H \\
y_{k} \in\{0,1\}, & \forall k \in H
\end{array}
$$

The objective function (6) maximizes the total flow captured by the follower. Constraint (7) determines the number of hubs to be located by the follower. Constraints (8) assure that the whole flow associated with each O/D pair is routed via some hub pair. Constraints (9) state that the flows can only be routed via nodes that have been designated as hubs. The constraints (10) and (11) define the real and binary variables, respectively.

\section{Tabu Search Algorithm}

The tabu search (TS) algorithm, proposed by Glover [11], is a local search algorithm successfully applied to a variety of hard optimization problems. The algorithm starts with an initial solution and uses a tabu list to control the search process in order to prevent trapping in local optima. From the current solution, all non-tabu moves are explored and the best one is selected. This move which might lead to an either better or worse solution than the current one, is recorded in the tabu list. The next move is among those not listed in the tabu list, unless it fulfills an aspiration level. TS would be terminated whenever a termination criterion such as a maximum number of iterations, or a fixed number of iterations with no improvement in the best solution, etc., is met.

\subsection{Solution Representation and Initial Solution Generation}

We use a one-dimensional array to represent the solutions and the size of this array is $r$ which includes the numbers associated with the nodes being selected as hubs by the follower. The sorting of numbers within the arrays is not important. Note that having known the selected hubs, for each $\mathrm{O} / \mathrm{D}$ pair $i-j$, one can easily determine the paths for routing the associated flow $w_{i j}$ by solving a shortest path problem.

To generate an initial solution, we randomly select $r$ out of $n$ nodes as hub nodes. This method for generating the initial solution is not only quick, but it also produces diverse solutions which can usually help the algorithm not to get trapped in local optima.

\subsection{Neighborhood Structures}

We define and use a single operator in our algorithm for generating neighbors. This operator, called "Swap", is used to alter one of the hubs in the solutions. To this end, we randomly select a hub node and a non-hub node. Then, if the selected non-hub node is not in the tabu list, the selected hub node becomes a non-hub node and the selected non-hub node becomes a hub.

\subsection{Parameters Used in the TS Algorithm}

The proposed TS algorithm uses two input parameters, namely $T L_{\text {size }}$ and $T_{\max } . T L_{\text {size }}$ represents the size of the tabu list and $T_{\max }$ denotes the number of iterations with no improvement in the best solution. The algorithm would be terminated when the algorithm does not improve the best solution in the last $T_{\max }$ iterations. 


\subsection{The Overall TS Algorithm}

To solve the $\left(r \mid X_{p}\right)$ hub-medianoid problem, as mentioned before, we assume that the leader has already located her hubs based on the solution of the uncapacitated multiple allocation $p$-hub median problem (UMApHLP) for multiple allocation network.

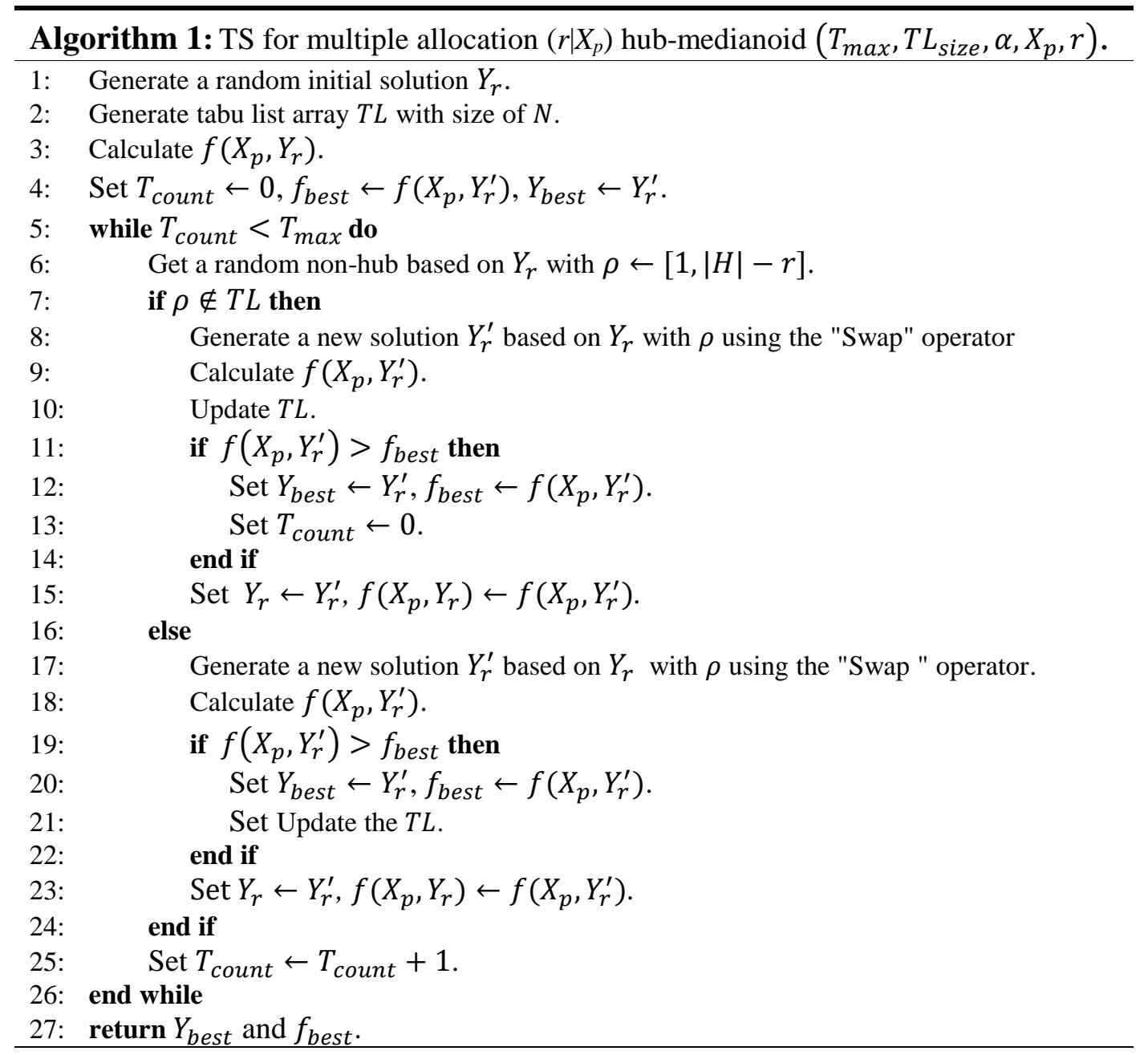

In the proposed TS algorithm, we set the size of the tabu list array $T L$ as $T L_{\text {size }}$, which is an input to the algorithm and also set a fixed number of iterations with no improvements in the best solution as $T_{\text {count }}$, which is input parameter. $Y_{\text {best }}$ indicates the best solution found so far and $f_{\text {best }}$ denotes the corresponding objective function value. In each iteration, we generate a random integer number $\rho$ on the interval $[0,|H|-r]$ to get a candidate non-hub. If $\rho \notin T L$, we generate a new solution $\left(Y_{r}^{\prime}\right)$ based on the current solution $\left(Y_{r}\right)$ using the "Swap" operator, which is defined previously. Subsequently, the objective function of the new solution, $\left(X_{p}, Y_{r}^{\prime}\right)$, is calculated and $T L$ is updated based on the first-in-first-out (FIFO) rule. If the objective function $f\left(X_{p}, Y_{r}^{\prime}\right)$ at the new solution $Y_{r}^{\prime}$ is even larger than the best objective value, $f_{\text {best }}$, so far, we set $Y_{\text {best }} \leftarrow Y_{r}^{\prime}, f_{\text {best }} \leftarrow f\left(X_{p}, Y_{r}^{\prime}\right)$ and $T_{\text {count }} \leftarrow 0$. In addition, we update the current solution, $Y_{r} \leftarrow Y_{r}^{\prime}$, and the current objective function, $f\left(X_{p}, Y_{r}\right) \leftarrow$ 
$f\left(X_{p}, Y_{r}^{\prime}\right)$. Otherwise, if $\rho \in T L$, we generate a new solution $Y_{r}^{\prime}$ using the "Swap" operator and calculate the objective function at the new solution. If $f\left(X_{p}, Y_{r}^{\prime}\right)>f_{\text {best }}$, we set $Y_{\text {best }} \leftarrow Y_{r}^{\prime}$ and $f_{\text {best }} \leftarrow f\left(X_{p}, Y_{r}^{\prime}\right)$ and update the tabu list $(T L)$. Moreover, we set the current objective function, $\left(X_{p}, Y_{r}\right) \leftarrow f\left(X_{p}, Y_{r}^{\prime}\right)$, and the current solution, $Y_{r} \leftarrow Y_{r}^{\prime}$. Subsequently, we add one to $T_{\text {count }}$. The algorithm is terminated when the number of iteration $T_{\text {count }}$ reaches the maxim fixed number of iterations with no improvement in the best solution $T_{\max }$. The pseudo-codes of the proposed TS algorithm for the multiple allocation $\left(r \mid X_{p}\right)$ hub-medianoid problem is shown in Algorithm 1.

\section{Computational Experiments}

In this section we present the results obtained from our computational experiments. Experiments are conducted in order to test the effectiveness of the proposed TS algorithm. To this end, we use two famous data sets from the literature of HLP: CAB and TR. The CAB data set introduced by O'Kelly [21] is based on the airline passenger interactions between 25 US cities in 1970 evaluated by the Civil Aeronautics Board $(\mathrm{CAB})$. This data set has been used by most of the hub location researchers in the literature in which all the 25 nodes are candidates for being hubs $(|H|=|N|=25)$. The second data set that is used in our computational experiments is the TR data set [28] which is based on the cargo flows between 81 cities $(|N|=81)$ on Turkey. Most authors use only 22 most populated cities as candidate nodes for locating hubs $(|H|=22)$. However, we also use the case where all the nodes are candidates for locating hubs $(|H|=81)$ as a large data set in our experiment.

We have generated and used 32 instances from the $\mathrm{CAB}$ data set and 171 instances from the TR data set. For the CAB data set, the number of hubs to be opened by the leader $(p)$ and the follower $(r)$ are selected as $r, p \in\{2,3,4,5\}$, and the parameter $\alpha$ is considered at two levels: 0.6 , and 0.8 . For the TR data set, two sets of instances are considered with small and large values as the number of hubs, respectively. In the first set of instances $(r, p \leq 5)$, the number of hubs are selected as $r, p \in\{2,3,4,5\}$, while in the second set $(r, p \geq 6)$, the number of hubs are selected as $r, p \in\{6,8,10,12,14\}$. In both cases, the parameter $\alpha$ is considered at three levels: $0.6,0.8$, and 0.9 as in [17].

The proposed TS algorithms are implemented in Microsoft Visual C\# 2013 (version 5.0). Also, the mathematical model for the follower problem is solved independently using the CPLEX version 12.6. All the experiments were run on a computer with Intel(R) Core(TM) Intel(R) Core(TM) i3-295 CPU of $3.30 \mathrm{GHz}$ and $16 \mathrm{~GB}$ of RAM, using the Microsoft Windows 7 operating system.

In a set of preliminary experiments, different combinations of algorithm parameters have been tested in order to tune their values so that high-quality solutions be obtained in short CPU times. Based on our findings, the tabu list size $\left(T L_{\text {size }}\right)$ is chosen as 10 and 7 for the CAB and TR data sets, respectively. Furthermore, we set the termination criterion for our algorithm to be 15 iterations with no improvement in the quality of best solution. Computational experiments were conducted using the above mentioned test problems to show the efficiency of the proposed TS algorithm and the results

are reported in the following section. For each problem instance, we ran the TS algorithm for three times and the best obtained solution is reported. 


\subsection{Numerical Results}

Table 1 shows the results obtained by solving the problem using the proposed TS algorithm as well as CPLEX based on the proposed mathematical model (6)-(11) with the CAB data set for different discount factors. Since the distance matrix in the CAB data set (also in the TR data set) is symmetric, it is clear that if the flow $w_{i j}$ from node $i \in N$ to node $j \in N$ is captured by the follower, the flow from node $j$ to node $i$ is also captured by the follower. Furthermore, since the flow from each node $i \in N$ to itself is zero (i.e., $w_{i i}=0$ ), in our computational studies the constraints (8)-(10) are imposed for only $i<j$ and the objective (6) is modified as

$$
\sum_{i \in N} \sum_{\substack{j \in N \\(j>i)}} \sum_{k \in H} \sum_{m \in H}\left(w_{i j}+w_{j i}\right) q_{i j k m} z_{i j k m},
$$

to reduce the size of our model. To set the location of the hubs in the incumbent leader's network, we assume that the leader has already located her hubs based on the uncapacitated multiple allocation $p$ hub median problem (UMApHMP). Therefore, before solving the follower's problem, we solve the leader's problem based on UMApHMP and obtain the optimal location for her hubs which are subsequently used in our numerical experimentations.

The columns entitled $p$ and $r$ denote the number of hubs which are opened by the leader and the follower, respectively. The next two columns show the follower's capture as the optimal objective function value obtained by CPLEX and the corresponding CPU time (in seconds). Finally, the two columns under the label "TS" give the best objective function value obtained by solving the instances with the TS algorithm and the average CPU time for the three runs of the TS algorithm.

As can be seen from the above Table 1, the proposed TS algorithm solves all instances to optimality within a fraction of a second. This is an indication of the efficiency of the proposed TS algorithm. Observe that the solution times for CPLEX are substantially larger than the corresponding times for the TS algorithm. Note also that since the leader decides on the location of her hubs based on a cost minimization criterion as in UMApHMP, which does not take into account the upcoming competition, the follower can capture a considerable share of the market upon entrance to the market. For example, when the follower locates the same number of hubs as the leader's, i.e., $p=r=2$, the market share of the follower is more than $65 \%$ of the total demand. 
Table 1. Results for solving the problems using the CAB data set

\begin{tabular}{|c|c|c|c|c|c|c|c|c|c|}
\hline \multirow{3}{*}{$p$} & \multirow{3}{*}{$r$} & \multicolumn{4}{|c|}{$\alpha=0.6$} & \multicolumn{4}{|c|}{$\alpha=0.8$} \\
\hline & & \multicolumn{2}{|c|}{ CPLEX } & \multicolumn{2}{|c|}{$\mathrm{TS}$} & \multicolumn{2}{|c|}{ CPLEX } & \multicolumn{2}{|c|}{$\mathrm{TS}$} \\
\hline & & $\begin{array}{l}\text { OF } \\
(\%)\end{array}$ & $\begin{array}{c}\mathrm{CPU} \\
\text { (s) }\end{array}$ & $\begin{array}{l}\mathrm{OF} \\
(\%)\end{array}$ & $\begin{array}{l}\text { CPU } \\
\text { (s) }\end{array}$ & $\begin{array}{l}\text { OF } \\
(\%)\end{array}$ & $\begin{array}{c}\mathrm{CPU} \\
\text { (s) }\end{array}$ & $\begin{array}{l}\text { OF } \\
(\%)\end{array}$ & $\begin{array}{l}\text { CPU } \\
\text { (s) }\end{array}$ \\
\hline \multirow[t]{4}{*}{2} & 2 & 65.62 & 14.74 & 65.62 & 0.02 & 65.84 & 8.82 & 65.84 & 0.03 \\
\hline & 3 & 78.25 & 17.56 & 78.25 & 0.06 & 74.19 & 22.03 & 74.19 & 0.05 \\
\hline & 4 & 87.08 & 17.83 & 87.08 & 0.07 & 80.69 & 25.10 & 80.69 & 0.10 \\
\hline & 5 & 92.38 & 16.05 & 92.38 & 0.10 & 87.14 & 18.35 & 87.14 & 0.11 \\
\hline \multirow[t]{4}{*}{3} & 2 & 30.49 & 26.60 & 30.49 & 0.06 & 29.18 & 24.99 & 29.18 & 0.05 \\
\hline & 3 & 45.13 & 24.56 & 45.13 & 0.08 & 42.92 & 22.58 & 42.92 & 0.06 \\
\hline & 4 & 53.69 & 22.13 & 53.69 & 0.12 & 52.83 & 20.57 & 52.83 & 0.11 \\
\hline & 5 & 62.02 & 23.93 & 62.02 & 0.15 & 60.14 & 22.47 & 60.14 & 0.13 \\
\hline \multirow[t]{4}{*}{4} & 2 & 18.89 & 27.72 & 18.89 & 0.06 & 21.06 & 24.48 & 21.06 & 0.05 \\
\hline & 3 & 28.39 & 30.68 & 28.39 & 0.09 & 32.69 & 21.92 & 32.69 & 0.06 \\
\hline & 4 & 37.73 & 33.16 & 37.73 & 0.20 & 42.10 & 23.47 & 42.10 & 0.13 \\
\hline & 5 & 46.18 & 25.76 & 46.18 & 0.22 & 48.60 & 25.25 & 48.60 & 0.14 \\
\hline \multirow[t]{4}{*}{5} & 2 & 18.64 & 27.97 & 18.64 & 0.08 & 18.19 & 23.49 & 18.19 & 0.08 \\
\hline & 3 & 28.14 & 23.62 & 28.14 & 0.09 & 29.12 & 20.11 & 29.12 & 0.14 \\
\hline & 4 & 35.04 & 19.22 & 35.04 & 0.19 & 36.93 & 24.00 & 36.93 & 0.11 \\
\hline & 5 & 42.32 & 22.77 & 42.32 & 0.16 & 44.32 & 25.48 & 44.32 & 0.13 \\
\hline \multicolumn{2}{|c|}{ Avg. } & 48.12 & 23.39 & 48.12 & 0.10 & 47.87 & 22.07 & 47.87 & 0.09 \\
\hline
\end{tabular}

Table 2 shows the results obtained by solving the problem using the TR data set for $r, p \leq 5$. Here, it is also assumed that the leader has already selected her $p$ hubs based on UMApHMP. Similar to the case of the $\mathrm{CAB}$ data set, to evaluate the performance of the proposed TS algorithm on the TR data set, all instances were solved to optimality using CPLEX and the results obtained by the TS are compared to their corresponding optimal values. 
Table 2. Results for solving the problem using the TR data set with $|H|=22(r, p \leq 5)$

\begin{tabular}{|c|c|c|c|c|c|c|c|c|c|c|c|c|c|}
\hline \multirow{3}{*}{$p$} & \multirow{3}{*}{$r$} & \multicolumn{4}{|c|}{$\alpha=0.6$} & \multicolumn{4}{|c|}{$\alpha=0.8$} & \multicolumn{4}{|c|}{$\alpha=0.9$} \\
\hline & & \multicolumn{2}{|c|}{ CPLEX } & \multicolumn{2}{|c|}{$\mathrm{TS}$} & \multicolumn{2}{|c|}{ CPLEX } & \multicolumn{2}{|c|}{$\mathrm{TS}$} & \multicolumn{2}{|c|}{ CPLEX } & \multicolumn{2}{|c|}{ TS } \\
\hline & & $\begin{array}{l}\mathrm{OF} \\
(\%)\end{array}$ & $\begin{array}{c}\mathrm{CPU} \\
\text { (s) }\end{array}$ & $\begin{array}{l}\text { OF } \\
(\%)\end{array}$ & $\begin{array}{c}\mathrm{CPU} \\
\text { (s) }\end{array}$ & $\begin{array}{l}\text { OF } \\
(\%)\end{array}$ & $\begin{array}{l}\mathrm{CPU} \\
\text { (s) }\end{array}$ & $\begin{array}{l}\text { OF } \\
(\%)\end{array}$ & $\begin{array}{l}\mathrm{CPU} \\
\text { (s) }\end{array}$ & $\begin{array}{l}\mathrm{OF} \\
(\%)\end{array}$ & $\begin{array}{l}\mathrm{CPU} \\
\text { (s) }\end{array}$ & $\begin{array}{l}\text { OF } \\
(\%)\end{array}$ & $\begin{array}{c}\mathrm{CPU} \\
\text { (s) }\end{array}$ \\
\hline \multirow{4}{*}{2} & 2 & 50.60 & 456.21 & 50.60 & 0.18 & 49.95 & 538.33 & 49.95 & 0.19 & 50.66 & 476.94 & 50.66 & 0.18 \\
\hline & 3 & 68.73 & 673.52 & 68.73 & 0.39 & 62.48 & 878.01 & 62.48 & 0.40 & 67.09 & 558.60 & 67.09 & 0.35 \\
\hline & 4 & 80.13 & 344.27 & 80.13 & 0.46 & 72.47 & 568.05 & 72.47 & 0.44 & 77.52 & 323.88 & 77.52 & 0.44 \\
\hline & 5 & 89.97 & 115.03 & 89.97 & 0.51 & 84.88 & 132.67 & 84.88 & 0.52 & 85.27 & 191.20 & 85.27 & 0.62 \\
\hline \multirow{4}{*}{3} & 2 & 30.49 & 1873.32 & 30.49 & 0.36 & 30.68 & 1435.06 & 30.68 & 0.37 & 40.58 & 233.93 & 40.58 & 0.35 \\
\hline & 3 & 40.82 & 1245.46 & 40.82 & 0.48 & 40.80 & 1667.72 & 40.80 & 0.43 & 52.71 & 494.27 & 52.71 & 0.51 \\
\hline & 4 & 56.40 & 577.22 & 56.40 & 0.60 & 51.43 & 959.99 & 51.43 & 0.69 & 63.24 & 360.14 & 63.24 & 0.72 \\
\hline & 5 & 66.43 & 487.51 & 66.43 & 0.84 & 60.66 & 560.07 & 60.66 & 0.81 & 72.38 & 130.33 & 72.38 & 0.92 \\
\hline \multirow{4}{*}{4} & 2 & 22.14 & 1389.16 & 22.14 & 0.55 & 20.33 & 2223.10 & 20.33 & 0.61 & 20.38 & 1465.93 & 20.38 & 0.69 \\
\hline & 3 & 33.69 & 739.71 & 33.69 & 0.82 & 30.18 & 1897.41 & 30.18 & 0.80 & 30.55 & 1777.43 & 30.55 & 0.81 \\
\hline & 4 & 44.79 & 949.52 & 44.79 & 0.91 & 39.40 & 1604.90 & 39.40 & 0.98 & 38.46 & 1316.08 & 38.46 & 0.97 \\
\hline & 5 & 55.69 & 517.45 & 55.69 & 1.09 & 48.57 & 786.07 & 48.57 & 1.22 & 47.40 & 565.69 & 47.40 & 1.18 \\
\hline \multirow{4}{*}{5} & 2 & 15.01 & 2076.17 & 15.01 & 0.78 & 15.72 & 1638.12 & 15.72 & 0.79 & 16.47 & 1387.98 & 16.47 & 0.91 \\
\hline & 3 & 23.88 & 1457.35 & 23.88 & 0.91 & 24.24 & 1618.06 & 24.24 & 0.95 & 23.94 & 1258.68 & 23.94 & 0.95 \\
\hline & 4 & 33.97 & 528.04 & 33.97 & 1.26 & 32.69 & 665.54 & 32.69 & 1.13 & 33.03 & 670.19 & 33.03 & 1.15 \\
\hline & 5 & 42.20 & 397.08 & 42.20 & 1.30 & 40.21 & 381.95 & 40.21 & 1.28 & 41.01 & 400.56 & 41.01 & 1.20 \\
\hline \multicolumn{2}{|c|}{ Avg. } & 47.18 & 864.19 & 47.18 & 0.71 & 44.04 & 1097.19 & 44.04 & 0.72 & 47.54 & 725.74 & 47.54 & 0.74 \\
\hline
\end{tabular}

The results on Table 2 reveal that the solution times by CPLEX substantially grow as the size of the problems increased. However, one can see that the proposed TS algorithm is able to obtain the optimal solutions for all the instances in the TR data set, with $r, p \leq 5$, within a fraction of a second, on the average. An interesting observation from this table is that in case of equal number of hubs for the leader and the follower, that is $p=r$, the leader captures more than half of the market share. In other words, the follower should open more hubs on order to capture more than half of the market share. Results obtained by solving the problems with the TR data sets for $r, p \geq 6$ are shown in Table 3. 
Table 3. Results for solving the problem for the TR data set with $|\boldsymbol{H}|=\mathbf{2 2}(\boldsymbol{r}, \boldsymbol{p} \geq \mathbf{6})$

\begin{tabular}{|c|c|c|c|c|c|c|c|c|c|c|c|c|c|}
\hline \multirow{3}{*}{$p$} & \multirow{3}{*}{$r$} & \multicolumn{4}{|c|}{$\alpha=0.6$} & \multicolumn{4}{|c|}{$\alpha=0.8$} & \multicolumn{4}{|c|}{$\alpha=0.9$} \\
\hline & & \multicolumn{2}{|c|}{ CPLEX } & \multicolumn{2}{|c|}{ TS } & \multicolumn{2}{|c|}{ CPLEX } & \multicolumn{2}{|c|}{$\mathrm{TS}$} & \multicolumn{2}{|c|}{ CPLEX } & \multicolumn{2}{|c|}{ TS } \\
\hline & & $\begin{array}{l}\mathrm{OF} \\
(\%)\end{array}$ & $\begin{array}{l}\mathrm{CPU} \\
\text { (s) }\end{array}$ & $\begin{array}{l}\mathrm{OF} \\
(\%)\end{array}$ & $\begin{array}{l}\mathrm{CPU} \\
\text { (s) }\end{array}$ & $\begin{array}{l}\mathrm{OF} \\
(\%)\end{array}$ & $\begin{array}{c}\mathrm{CPU} \\
(\mathrm{s})\end{array}$ & $\begin{array}{l}\mathrm{OF} \\
(\%)\end{array}$ & $\begin{array}{c}\mathrm{CPU} \\
\text { (s) }\end{array}$ & $\begin{array}{l}\mathrm{OF} \\
(\%)\end{array}$ & $\begin{array}{c}\text { CPU } \\
(\mathrm{s})\end{array}$ & $\begin{array}{l}\mathrm{OF} \\
(\%)\end{array}$ & $\begin{array}{c}\mathrm{CPU} \\
\text { (s) }\end{array}$ \\
\hline \multirow[t]{5}{*}{6} & 6 & 39.31 & 330.68 & 39.31 & 2.43 & 37.97 & 329.58 & 37.97 & 3.16 & 40.86 & 199.74 & 40.86 & 3.89 \\
\hline & 8 & 49.19 & 213.08 & 49.19 & 3.59 & 48.24 & 210.68 & 48.24 & 3.08 & 49.44 & 208.29 & 49.44 & 2.75 \\
\hline & 10 & 56.94 & 241.03 & 56.94 & 3.36 & 55.70 & 156.08 & 55.70 & 2.59 & 56.04 & 140.39 & 56.04 & 3.15 \\
\hline & 12 & 64.02 & 89.82 & 64.02 & 4.76 & 61.84 & 104.83 & 61.84 & 4.15 & 61.54 & 110.19 & 61.54 & 4.10 \\
\hline & 14 & 68.91 & 46.73 & 68.91 & 4.50 & 66.97 & 49.58 & 66.97 & 4.31 & 66.45 & 63.93 & 66.45 & 5.10 \\
\hline \multirow[t]{5}{*}{8} & 6 & 28.58 & 311.06 & 28.58 & 3.27 & 29.37 & 298.18 & 29.37 & 3.41 & 31.10 & 162.97 & 31.10 & 3.41 \\
\hline & 8 & 37.09 & 311.12 & 37.09 & 5.17 & 37.08 & 188.41 & 37.08 & 4.82 & 38.69 & 178.41 & 38.69 & 5.04 \\
\hline & 10 & 44.37 & 188.64 & 44.37 & 4.33 & 44.35 & 120.10 & 44.35 & 4.30 & 44.83 & 141.54 & 44.83 & 4.87 \\
\hline & 12 & 51.77 & 104.74 & 51.77 & 3.12 & 50.71 & 87.52 & 50.71 & 3.40 & 50.49 & 65.47 & 50.49 & 4.13 \\
\hline & 14 & 57.97 & 32.70 & 57.97 & 2.51 & 56.33 & 57.38 & 56.33 & 3.17 & 55.77 & 55.71 & 55.77 & 2.98 \\
\hline \multirow[t]{5}{*}{10} & 6 & 19.91 & 239.45 & 19.91 & 3.87 & 20.12 & 212.75 & 20.12 & 3.87 & 20.74 & 198.06 & 20.74 & 3.75 \\
\hline & 8 & 27.13 & 163.11 & 27.13 & 2.57 & 27.03 & 130.36 & 27.03 & 2.57 & 27.77 & 119.45 & 27.77 & 2.41 \\
\hline & 10 & 34.10 & 119.31 & 34.10 & 3.49 & 33.84 & 102.23 & 33.84 & 3.49 & 33.86 & 88.82 & 33.86 & 4.19 \\
\hline & 12 & 40.48 & 65.47 & 40.48 & 3.14 & 40.74 & 58.40 & 40.74 & 3.14 & 39.89 & 57.53 & 39.89 & 3.39 \\
\hline & 14 & 45.73 & 42.69 & 45.73 & 4.15 & 46.84 & 29.62 & 46.84 & 4.15 & 44.90 & 30.97 & 44.90 & 4.72 \\
\hline \multirow[t]{5}{*}{12} & 6 & 15.83 & 121.95 & 15.83 & 3.92 & 16.93 & 79.40 & 16.93 & 3.92 & 18.45 & 72.18 & 18.45 & 4.05 \\
\hline & 8 & 21.79 & 79.38 & 21.79 & 4.39 & 23.41 & 53.09 & 23.41 & 4.39 & 24.59 & 32.61 & 24.59 & 3.98 \\
\hline & 10 & 27.06 & 55.82 & 27.06 & 5.44 & 28.62 & 31.82 & 28.62 & 5.44 & 29.08 & 21.75 & 29.08 & 6.15 \\
\hline & 12 & 31.37 & 41.83 & 31.37 & 5.32 & 32.81 & 22.33 & 32.81 & 5.32 & 32.98 & 23.53 & 32.98 & 5.09 \\
\hline & 14 & 35.48 & 32.71 & 35.48 & 3.42 & 35.93 & 20.83 & 35.93 & 3.42 & 36.18 & 18.40 & 36.18 & 3.86 \\
\hline \multirow[t]{5}{*}{14} & 6 & 13.04 & 58.69 & 13.04 & 7.79 & 13.02 & 59.85 & 13.02 & 7.79 & 13.66 & 57.79 & 13.66 & 6.74 \\
\hline & 8 & 17.87 & 54.39 & 17.87 & 4.89 & 18.57 & 20.04 & 18.57 & 4.89 & 18.81 & 19.43 & 18.81 & 5.15 \\
\hline & 10 & 22.25 & 37.53 & 22.25 & 6.84 & 22.52 & 20.16 & 22.52 & 6.84 & 22.50 & 18.87 & 22.50 & 6.85 \\
\hline & 12 & 26.00 & 19.86 & 26.00 & 4.60 & 25.20 & 18.22 & 25.20 & 4.60 & 25.60 & 22.26 & 25.60 & 5.16 \\
\hline & 14 & 28.42 & 20.22 & 28.42 & 5.07 & 27.46 & 18.89 & 27.46 & 5.07 & 28.18 & 17.27 & 28.18 & 4.75 \\
\hline \multicolumn{2}{|c|}{ Avg. } & 36.18 & 120.88 & 36.18 & 4.23 & 36.06 & 99.21 & 36.06 & 4.21 & 36.50 & 85.02 & 36.50 & 4.38 \\
\hline
\end{tabular}

We can observe from Table 3 that the TS algorithm is able to obtain the optimal solutions of all the instances of the TR data set with $r, p \geq 6$ The average solution time for these instances is less than 5 seconds on which shows the high efficiency of the proposed solution algorithm. An interesting observation from the above tables is that CPLEX solves the TR instances with $r, p \geq 6$ in shorter time than the instances with $r, p \leq 5$ This can be due to the limited number of candidate nodes for hub $(|H|=22)$ in the above experiments. In this case, as the number of hubs located by the leader gets larger, the set of promising solutions for the follower becomes smaller and hence the time taken to reach the optimal solution by CPLEX gets shorter. Comparing the results for different values of discount factor, one can see that the capture values are similar for different values. This is because of the fact that the same discount factor applies for both firms. Another important observation form the above tables is that as the number of hubs opened by the leader $(p)$ increases, the follower fails to capture much of the market share even if $r>p$. One possible reason for this can be the fact that as $p$ increases, the leader selects more of the critical locations for opening hubs and increases her provided service level. In addition, since the customers choose the leader's service on case of equal service offered by the leader and the follower, the leader's market share stays higher than that of the follower. 
The efficiency and advantage of the proposed TS algorithm is more evident when it is shown that it solves large instances which are not solvable within reasonable times by the commercial solvers. To this end, our next set of experiments is conducted on a larger data set by assuming that all the 81 nodes in the TR data set are available as candidate locations for installing hub facilities (i.e., $|H|=81$ ) by both the leader and the follower. Initially, we tried to solve the instances using CPLEX. However, due to the large size of these instances (as it now includes all the 81 nodes of the TR data set as candidate locations for installing hubs), CPLEX was not able to solve any of the instances within the time limit of 5 hours. Therefore, to evaluate the efficiency of the proposed TS algorithm, we solved the problem using an existing simulated annealing (SA) based algorithm proposed in [10]. The results for solving the problem using the TS and SA algorithms with the TR data set by assuming $|H|=81$ are reported in Table 4. It is assumed that the leader has already selected its $p$ hubs based on UMApHMP where all the 81 nodes are candidates for being hub. Since CPLEX was not able to solve any of the instances within the time limit of 5 hours, no result is not reported for its performance.

Table 4. Results for the problems of the TR data set with $|H|=81(r, p \leq 5)$

\begin{tabular}{|c|c|c|c|c|c|c|c|c|c|c|c|c|c|}
\hline \multirow{3}{*}{$p$} & \multirow{3}{*}{$r$} & \multicolumn{4}{|c|}{$\alpha=0.6$} & \multicolumn{4}{|c|}{$\alpha=0.8$} & \multicolumn{4}{|c|}{$\alpha=0.9$} \\
\hline & & \multicolumn{2}{|c|}{ SA [10] } & \multicolumn{2}{|c|}{$\mathrm{TS}$} & \multicolumn{2}{|c|}{ SA [10] } & \multicolumn{2}{|c|}{ TS } & \multicolumn{2}{|c|}{ SA [10] } & \multicolumn{2}{|c|}{ TS } \\
\hline & & $\begin{array}{l}\mathrm{OF} \\
(\%)\end{array}$ & $\begin{array}{c}\mathrm{CPU} \\
\text { (s) }\end{array}$ & $\begin{array}{l}\text { OF } \\
(\%)\end{array}$ & $\begin{array}{c}\mathrm{CPU} \\
\text { (s) }\end{array}$ & $\begin{array}{l}\text { OF } \\
(\%) \\
\end{array}$ & $\begin{array}{c}\text { CPU } \\
\text { (s) }\end{array}$ & $\begin{array}{l}\text { OF } \\
(\%)\end{array}$ & $\begin{array}{c}\mathrm{CPU} \\
\text { (s) }\end{array}$ & $\begin{array}{l}\text { OF } \\
(\%)\end{array}$ & $\begin{array}{l}\mathrm{CPU} \\
\text { (s) }\end{array}$ & $\begin{array}{l}\text { OF } \\
(\%)\end{array}$ & $\begin{array}{l}\mathrm{CPU} \\
\text { (s) }\end{array}$ \\
\hline \multirow[t]{4}{*}{2} & 2 & 43.44 & 0.83 & 44.40 & 0.31 & 43.55 & 0.89 & 43.55 & 0.29 & 42.08 & 0.77 & 42.08 & 0.33 \\
\hline & 3 & 67.00 & 1.12 & 67.00 & 0.49 & 61.99 & 1.05 & 61.99 & 0.50 & 57.44 & 1.08 & 58.09 & 0.54 \\
\hline & 4 & 79.86 & 1.60 & 79.86 & 0.65 & 74.05 & 1.63 & 74.05 & 0.59 & 72.56 & 1.76 & 72.56 & 0.64 \\
\hline & 5 & 87.71 & 2.32 & 87.71 & 1.02 & 83.49 & 2.29 & 83.49 & 0.94 & 80.54 & 2.40 & 80.54 & 0.98 \\
\hline \multirow[t]{4}{*}{3} & 2 & 27.29 & 1.12 & 30.15 & 0.40 & 28.55 & 1.13 & 28.55 & 0.41 & 28.56 & 1.10 & 28.56 & 0.47 \\
\hline & 3 & 44.60 & 1.50 & 44.60 & 0.53 & 42.36 & 1.45 & 42.36 & 0.59 & 38.73 & 1.39 & 41.40 & 0.64 \\
\hline & 4 & 59.83 & 1.91 & 59.83 & 0.68 & 54.80 & 1.89 & 54.80 & 0.66 & 53.60 & 1.90 & 53.60 & 0.73 \\
\hline & 5 & 73.67 & 2.42 & 73.67 & 1.05 & 65.31 & 2.41 & 65.31 & 1.02 & 63.84 & 2.59 & 63.84 & 1.07 \\
\hline \multirow[t]{4}{*}{4} & 2 & 22.81 & 1.56 & 22.81 & 0.40 & 26.63 & 1.69 & 27.33 & 0.44 & 26.58 & 1.62 & 26.58 & 0.51 \\
\hline & 3 & 34.50 & 1.74 & 34.50 & 0.55 & 37.08 & 1.80 & 37.08 & 0.59 & 36.11 & 1.88 & 37.99 & 0.65 \\
\hline & 4 & 46.24 & 2.10 & 46.24 & 0.60 & 46.97 & 2.09 & 46.97 & 0.67 & 49.89 & 2.10 & 49.89 & 0.59 \\
\hline & 5 & 59.92 & 2.61 & 59.92 & 1.12 & 56.08 & 2.75 & 56.08 & 1.10 & 58.20 & 2.84 & 58.20 & 1.14 \\
\hline \multirow[t]{4}{*}{5} & 2 & 16.19 & 2.16 & 16.50 & 0.49 & 15.57 & 2.17 & 15.57 & 0.50 & 15.70 & 2.15 & 15.70 & 0.51 \\
\hline & 3 & 24.85 & 2.60 & 24.85 & 0.60 & 27.64 & 2.67 & 27.64 & 0.61 & 24.57 & 2.52 & 24.57 & 0.69 \\
\hline & 4 & 34.80 & 2.98 & 34.80 & 0.84 & 36.11 & 3.01 & 36.11 & 0.93 & 35.49 & 3.09 & 35.49 & 0.91 \\
\hline & 5 & 44.63 & 3.47 & 44.63 & 1.19 & 45.36 & 3.45 & 45.36 & 1.15 & 45.19 & 3.39 & 45.19 & 1.18 \\
\hline \multicolumn{2}{|c|}{ Avg. } & 47.96 & 2.00 & 48.22 & 0.68 & 46.60 & 2.02 & 46.64 & 0.69 & 45.57 & 2.04 & 45.89 & 0.72 \\
\hline
\end{tabular}

The results in Table 4 show that the proposed TS algorithm outperforms the SA [10] algorithm both in terms of solution time and quality. It can be seen that for seven instances (denoted in bold in Table 4), the objective values of the solutions found by the TS are better than those of the SA's solution. For the rest of the instances, on the other hand, the two algorithms found the same solutions. Furthermore, the solution times for the TS are significantly smaller than those of the SA algorithm. These results show that the TS algorithm is very efficient in solving the competitive multiple allocation hub location problem. 


\subsection{Optimal Hub Locations on Map}

As the last part of our computational experiments, we illustrate how the decisions regarding the locations of the hubs alter for different values of discount factor. The analysis is conducted with the the $\mathrm{CAB}$ data set. Fig 2 depicts the optimal locations of the hubs opened by the leader and the follower, where $p=2, r=5$ and $\alpha=0.6$. The triangles show the locations of leader's hubs, whereas the squares show the optimal locations of the follower's hubs which are optimally located according to the locations chosen by the leader. As can be seen from Table 1, in this case the follower can capture $92.38 \%$ of the market share after locating its five hubs. One reason for such a large capture is the large number of hubs located by the follower as compared to the number of hubs located by the leader. Another reason is that the leader has located her hubs without considering the upcoming follower's entrance to the market. In other words, the leader could have improved her market share if the initial locations of her hubs were selected in anticipation of upcoming follower's entrance to the market.

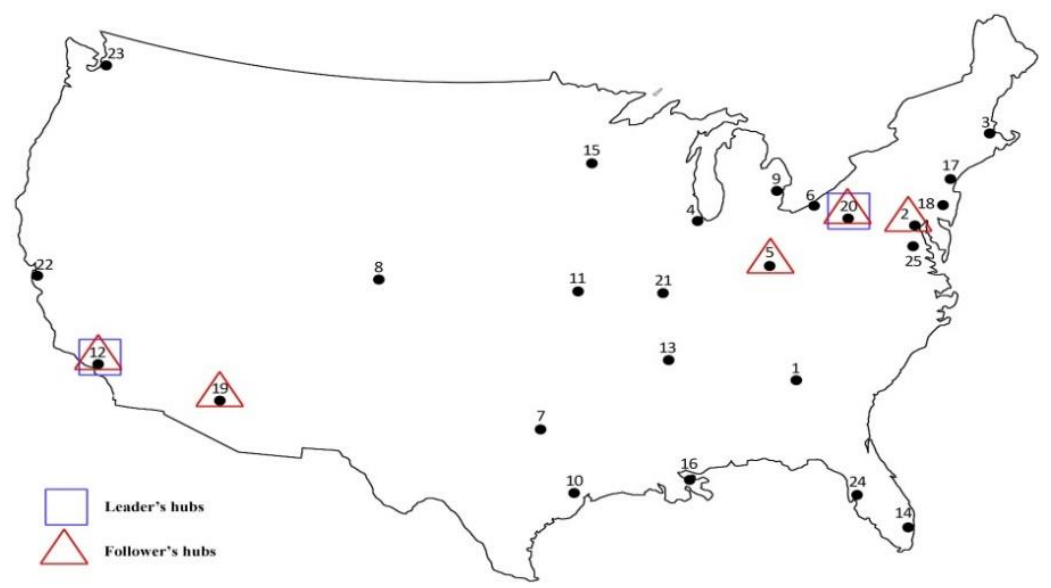

Figure 2. Optimal hub locations for the leader and the follower with $p=2, r=5$ and $\alpha=0.6$

Fig 3 depicts the optimal locations of the hubs opened by the leader and the follower for the $\mathrm{CAB}$ data set where $p=2, r=5$ and $\alpha=0.8$. Observe that the follower's optimal hub locations when the discount factor increased are different from the hub locations when $\alpha=0.6$. On the other hands, by increasing the discount factor $\alpha$ to 0.8 , the market share captured by the follower dropped to $87.14 \%$.

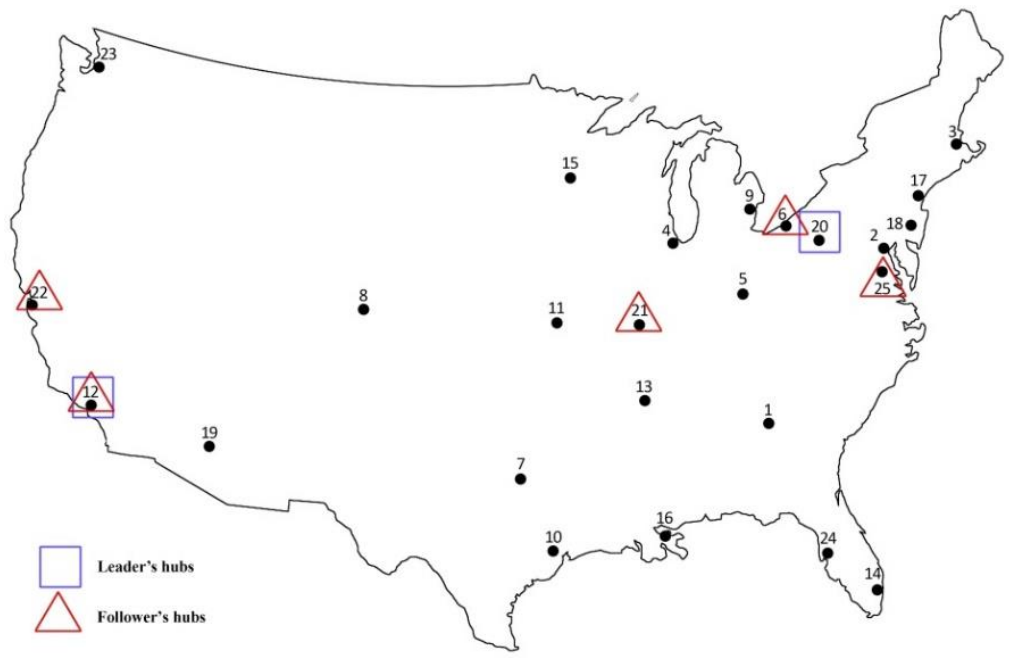

Figure 3. Optimal hub locations for the leader and the follower with $p=2, r=5$ and $\alpha=0.8$ 
It can be seen from the above maps that the hubs opened by the leader and the follower are different for $\alpha=0.6$ and $\alpha=0.8$, which can be regarded as a reason why variant levels of discount factor $\alpha$ need to be considered in computational experiments.

\section{Conclusions}

In this research, we considered the competitive multiple allocation HLP in a duopoly market. It was assumed that an incumbent firm (the leader) was operating an existing hub network in a market and an entrant firm (the follower) tried to enter the market by configuring its own hub network trying to capture as much flow as possible from the leader. The customers chose one firm based on the service level (cost, time, distance, etc.) provided by these firms. Therefore, the follower aimed at locating its hubs in such a way that its total captured flow (market share) was maximized. We proposed a mathematical formulation the follower's problem and an efficient tabu search (TS) based heuristic for solving the problem. Extensive computational experiments based on two data sets of the $\mathrm{CAB}$ and TR were conducted to analyze different properties of these problems and to evaluate the performance of the proposed TS algorithm as well as the mathematical model. In all the instances for the two data sets, the proposed TS algorithm obtained the optimal solutions. Furthermore, the computational results showed the efficiency of the proposed algorithm in terms of CPU times.

As an interesting line for future research, one can incorporate the leader's decisions to the problem and study a more comprehensive and realistic problem in which the leader and follower make decisions in a sequential game which can be modelled as a bilevel programming problem. Moreover, instead of assuming an all-or-nothing (binary) mechanism for the capture of flows by the players, one can use a more general mechanism such as partial capture may better reflect the typical problems in real-world contexts.

\section{References}

[1] Alumur, S., and Kara, B.Y. (2008), Network hub location problems: The state of the art, European journal of operational research, 190(1), 1-21.

[2] Campbell, J.F. (1994), Integer programming formulations of discrete hub location problems, European Journal of Operational Research, 72(2), 387-405.

[3] Campbell, J.F., and O'Kelly, M.E. (2012), Twenty-five years of hub location research, Transportation Science, 46(2), 153-169.

[4] Chen, J.F. (2007), A hybrid heuristic for the uncapacitated single allocation hub location problem, Omega, 35(2), 211-220.

[5] Čvokić, D.D., Kochetov, Y.A., and Plyasunov, A.V. (2016), A Leader-Follower Hub Location Problem Under Fixed Markups, In International Conference on Discrete Optimization and Operations Research (pp. 350-363), Springer International Publishing.

[6] Čvokić, D.D., Kochetov, Y.A., and Plyasunov, A. V. (2017), The Existence of Equilibria in the Leader-Follower Hub Location and Pricing Problem, In Operations Research Proceedings 2015 (pp. 539-544), Springer, Cham.

[7] Eiselt, H.A., and Marianov, V. (2009), A conditional p-hub location problem with attraction functions, Computers \& Operations Research, 36(12), 3128-3135.

[8] Ernst, A.T., and Krishnamoorthy, M. (1996), Efficient algorithms for the uncapacitated single allocation p-hub median problem, Location science, 4(3), 139-154. 
[9] Farahani, R.Z., Hekmatfar, M., Arabani, A.B., and Nikbakhsh, E. (2013), Hub location problems: A review of models, classification, solution techniques, and applications, Computers \& Industrial Engineering, 64(4), 1096-1109.

[10] Ghaffarinasab, N., Motallebzadeh, A., Jabarzadeh, Y., and Kara, B.Y. (2017), Efficient simulated annealing based solution approaches to the competitive single and multiple allocation hub location problems, Computers \& Operations Research (In Press), DOI: 10.1016/j.cor.2017.09.022.

[11] Glover, F. (1986), Future paths for integer programming and links to artificial intelligence, Computers \& Operations Research, 13(5), 533-549.

[12] Jabalameli, M.S., Barzinpour, F., Saboury, A., and Ghaffari-Nasab, N. (2012), A simulated annealing-based heuristic for the single allocation maximal covering hub location problem, International Journal of Metaheuristics, 2(1), 15-37.

[13] Kara, B.Y., and Tansel, B.C. (2003), The single-assignment hub covering problem: Models and linearizations, Journal of the Operational Research Society, 54(1), 59-64.

[14] Karimi, H., Bashiri, M., and Nickel, S. (2016), Capacitated single allocation p-hub covering problem in multi-modal network using tabu search, International Journal of EngineeringTransactions C: Aspects, 29(6), 797.

[15] Lin, C.C., and Lee, S.C. (2010), The competition game on hub network design, Transportation Research Part B: Methodological, 44(4), 618-629.

[16] Lüer-Villagra, A., and Marianov, V. (2013), A competitive hub location and pricing problem, European Journal of Operational Research, 231(3), 734-744.

[17] Mahmutogullari, A.I., and Kara, B.Y. (2016), Hub location under competition, European Journal of Operational Research, 250(1), 214-225.

[18] Marianov, V., Serra, D., and ReVelle, C. (1999), Location of hubs in a competitive environment, European Journal of Operational Research, 114(2), 363-371.

[19] Niknamfar, A.H., Niaki, S.T.A., and Niaki, S.A.A. (2017), Opposition-based learning for competitive hub location: A bi-objective biogeography-based optimization algorithm, Knowledge-Based Systems, 128, 1-19.

[20] O'kelly, M.E. (1986), The location of interacting hub facilities, Transportation science, 20(2), 92-106.

[21] O'kelly, M.E. (1987), A quadratic integer program for the location of interacting hub facilities, European Journal of Operational Research, 32(3), 393-404

[22] Pasandideh, S.H.R., Niaki, S.T.A., and Sheikhi, M. (2016), A bi-objective hub maximal covering location problem considering time-dependent reliability and the second type of coverage, International Journal of Management Science and Engineering Management, 11(4), 195-202.

[23] Peker, M., and Kara, B.Y. (2015), The P-Hub maximal covering problem and extensions for gradual decay functions, Omega, 54, 158-172.

[24] Sasaki, M., Campbell, J.F., Krishnamoorthy, M., and Ernst, A.T. (2014), A Stackelberg hub arc location model for a competitive environment, Computers \& Operations Research, 47, 27-41.

[25] Silva, M.R., and Cunha, C.B. (2009), New simple and efficient heuristics for the uncapacitated single allocation hub location problem, Computers \& Operations Research, 36(12), 3152-3165.

[26] Silva, M.R., and Cunha, C.B. (2017), A tabu search heuristic for the uncapacitated single allocation p-hub maximal covering problem, European Journal of Operational Research.

[27] Skorin-Kapov, D., and Skorin-Kapov, J. (1994), On tabu search for the location of interacting hub facilities, European Journal of Operational Research, 73(3), 502-509.

[28] Tan, P.Z., and Kara, B.Y. (2007), A hub covering model for cargo delivery systems, Networks, 49(1), 28-39. 
[29] Topcuoglu, H., Corut, F., Ermis, M., and Yilmaz, G. (2005), Solving the uncapacitated hub location problem using genetic algorithms, Computers \& Operations Research, 32(4), 967984.

[30] Wagner, B. (2008), A note on "location of hubs in a competitive environment", European Journal of Operational Research, 184(1), 57-62.

[31] Wagner, B. (2008), Model formulations for hub covering problems, Journal of the Operational Research Society, 59(7), 932-938.

[32] Yildız, B., and Karaşan, O. E. (2015), Regenerator Location Problem and survivable extensions: A hub covering location perspective, Transportation Research Part B: Methodological, 71, 32-55. 